\title{
Gastric Adenocarcinoma with Systemic Metastasis Involving the Intraocular Choroid and Duodenum
}

\author{
Soon Young Kim ${ }^{1}$, Kee-Taek Jang ${ }^{2}$ and Jun Haeng Lee ${ }^{1}$ \\ Departments of ${ }^{1}$ Medicine, ${ }^{2}$ Pathology and Translational Genomics, Samsung Medical Center, Sungkyunkwan University School of Medicine, \\ Seoul, Korea
}

Gastric cancer with double metastasis to the orbit and duodenum is extremely rare. We report the case of a patient with gastric adenocarcinoma who presented with synchronous orbital and duodenal metastases at the time of initial diagnosis. A 60-year-old man presented with a 1-month history of visual disorder and pain in his right eye. He underwent ophthalmological examinations. The biopsy results suggested intraocular metastatic carcinoma. We conducted a systemic evaluation to identify primary malignancy. Finally, a diagnosis of advanced gastric adenocarcinoma with multi-organ metastasis was made. He planned to be treated with systemic chemotherapy. Clin Endosc 2018;51:95-98

Key Words: Stomach neoplasms; Orbital metastasis; Duodenal metastasis

\section{INTRODUCTION}

Gastric cancer is a leading cause of cancer death worldwide. The most common metastatic sites of gastric cancer are the liver (40\%) and peritoneum. Sometimes, it metastasizes to periumbilical lymph nodes, left supraclavicular sentinel nodes, the pouch of Douglass, and the ovaries. ${ }^{1}$ Gastric cancer with orbital metastasis is rare, and so is that with accompanying duodenal metastasis. In Korea, 3 cases of orbital metastasis have been reported, ${ }^{2-4}$ but duodenal metastasis has never been reported. We report a patient with gastric adenocarcinoma who presented with orbital and duodenal metastases at the time of initial diagnosis.

Received: March 20, 2017 Revised: April 24, 2017

Accepted: April 26, 2017

Correspondence: Jun Haeng Lee

Department of Medicine, Samsung Medical Center, Sungkyunkwan University School of Medicine, 81 Irwon-ro, Gangnam-gu, Seoul 06351, Korea

Tel: +82-2-3410-3409, Fax: +82-2-3410-6983, E-mail: stomachlee@gmail.com

(cc) This is an Open Access article distributed under the terms of the Creative Commons Attribution Non-Commercial License (http://creativecommons.org/ licenses/by-nc/3.0) which permits unrestricted non-commercial use, distribution, and reproduction in any medium, provided the original work is properly cited.

\section{CASE REPORT}

A 60-year-old man was referred to the department of ophthalmology in our hospital because of a 1-month history of visual disorder and pain in his right eye. On presentation, the visual acuity of his right and left eyes were 0.05 and 0.9 , respectively. Slit-lamp biomicroscopy revealed an inferonasal choroidal mass in the right eye. The anterior segment examination result was normal for both eyes. The intraocular pressure was within its normal limits. Fundoscopic examination disclosed an elevated choroidal tumor infiltrating the macular area. An exudative retinal detachment involving the macula was found. Echography revealed a polygonal mass with heterogeneously strong internal echoes in the same region. A computed tomography (CT) scan of the orbits demonstrated a 1.5$\mathrm{cm}$ enhancing mass at the medial side of the choroid of the right eyeball. Magnetic resonance imaging (MRI) revealed the same lesion as an isointense signal on the T1-weighted image and as a hypointense signal on the T2-weighted image (Fig. 1). The diagnostic impression was a metastatic lesion. The left eye was unremarkable.

To identify the primary site of the metastatic cancer, we performed chest and abdominal CT. Radiological examination revealed advanced gastric cancer with central ulceration 
at the lesser curvature side of the high body. Multiple enlarged lymph nodes were observed at the perigastric, paraaortic, and mediastinal areas. Multiple liver, lung, and bone metastases were identified with peritoneal thickening.

18F-Fluorodeoxyglucose-positron emission tomography/ computed tomography (18-FDG-PET/CT) revealed hypermetabolic lesions in the gastric high body and antrum. The SUVmax values of the high body and antrum were 8.3 and 4.3, respectively. Multiple hypermetabolic lesions were observed in supraclavicular/intra-abdominal nodes, the liver, the right eyeball, and bones. The SUVmax of the right eyeball was 3.8, which implied a metastasis (Fig. 2).
We performed esophagogastroduodenoscopy, which revealed a 3-cm ulceroinfiltrative advanced gastric cancer at the lesser curvature of the cardia. Three round elevated lesions were observed at the duodenal second portion. Endoscopy showed no mucosal lesion at the antrum (Fig. 3). Endoscopic biopsies of the lesions were performed, respectively. Pathological examination disclosed a malignancy of moderately differentiated tubular adenocarcinoma (Fig. 4A). The lesion at the duodenum showed a poorly differentiated tubular adenocarcinoma (Fig. 4B). Immunohistochemistry staining for c-erbB-2 (HER2) was negative. Finally, we made a diagnosis of advanced gastric cancer with multiple metastasis. The patient
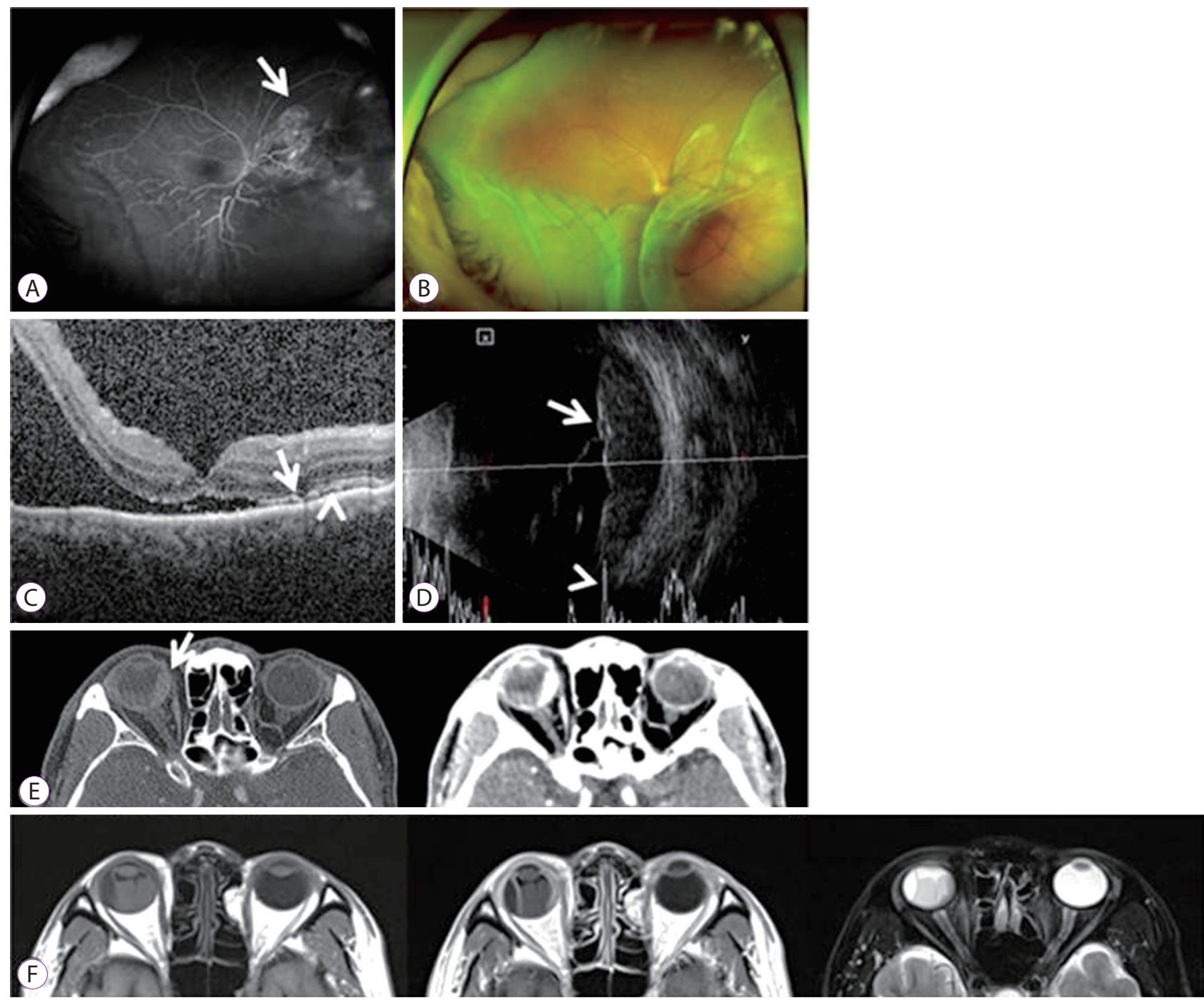

Fig. 1. (A) Wide-field fluorescein angiogram showing hyperfluorescence in the late venous phases with leakage at the tumor border (arrow). (B) Wide-field fundus photograph showing a choroidal mass with associated exudative retinal detachment at the inferior area, without abnormal pigmentation. (C) Optical coherence tomography scan showing shaggy photoreceptors (arrow), "lumpy-bumpy" appearance of a retinal pigment epithelium (arrowhead), and retinal detachment with subretinal fluid, involving the fovea center. (D) Ultrasonography scan showing a polygonal choroidal mass with solid consistency, $22.8 \times 5.5 \mathrm{~mm}$ in size, on A-scan (arrow). The B-scan shows high irregular reflectivity (arrowhead). (E) Computed tomography scan showing wall thickening of the medial side of the right eye (arrow), with enhancement. (F) Magnetic resonance imaging scan showing wall thickening of the medial side of the right eye. The T1-weighted image shows isointensity with enhancement, and the T2-weighted image shows hypointensity. 

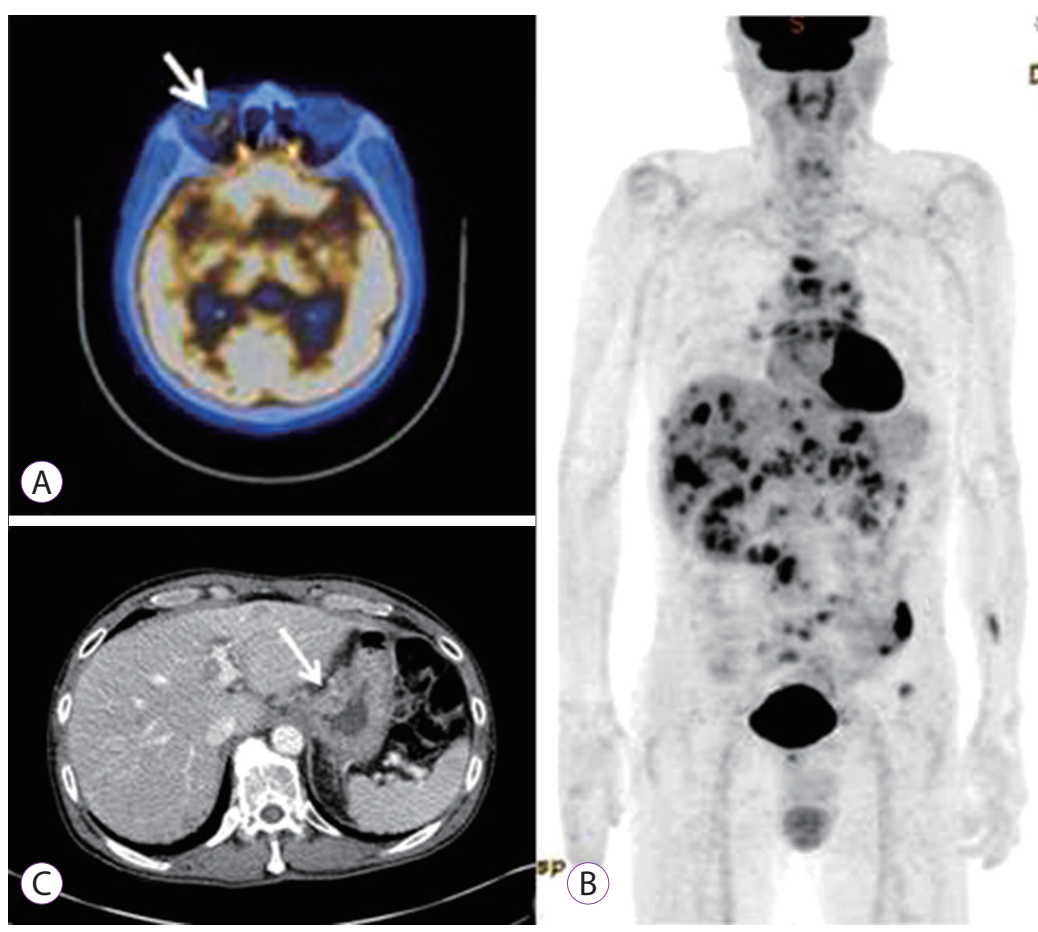

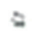

D.

Fig. 2. (A) 18F-Fluorodeoxyglucose-positron emission tomography (18-FDG-PET) scan showing increased FDG uptake at the right eyeball (arrow). (B) 18-FDG-PET scan showing multiple hypermetabolic lesions in the stomach, lymph nodes, liver, and bone. (C) Computed tomography scan showing advanced gastric cancer with ulceration at the cardia (arrow).
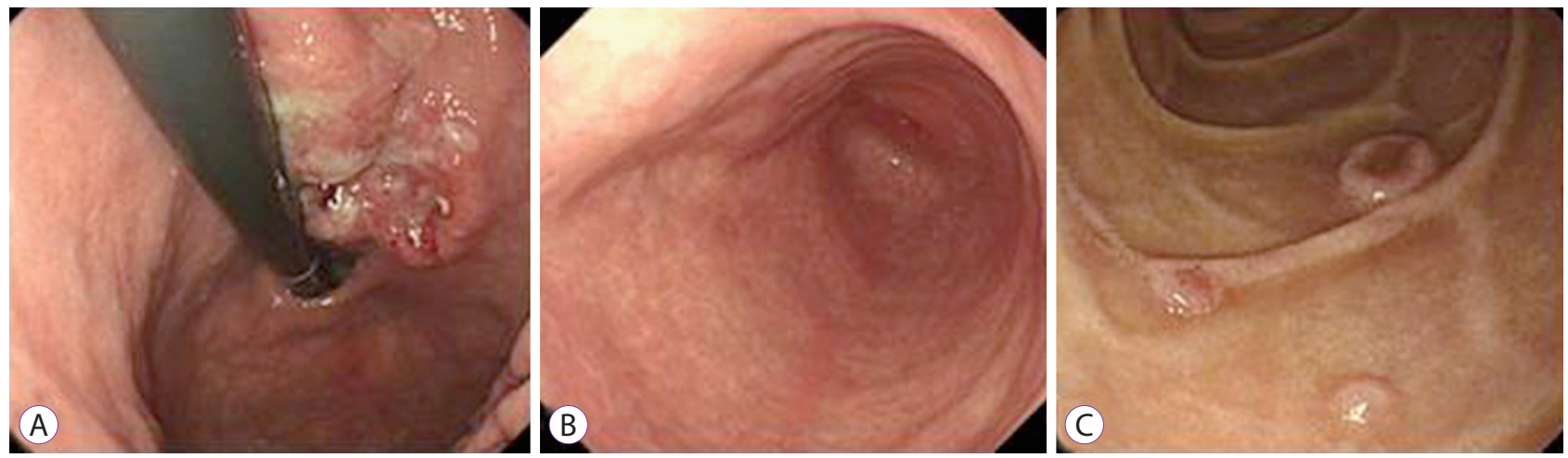

Fig. 3. Esophagogastroduodenoscopy image. (A) An ulceroinfiltrative gastric cancer, $3 \mathrm{~cm}$ in size, at the lesser curvature side of the cardia. (B) No mucosal lesion on the antrum. (C) Three slightly elevated round lesions on the second portion of the duodenum.

started receiving chemotherapy with intravenous oxaliplatin and oral capecitabine.

\section{DISCUSSION}

Intraocular malignancy is a rare disease. Metastatic tumor is the most common type of intraocular malignancy. ${ }^{5}$ Nevertheless, metastatic tumor to the orbit accounts for $2 \%$ to $3 \%$ of all systemic cancers. ${ }^{6}$ The representative symptoms of ocular metastasis are pain, exophthalmos, and decreased vision. ${ }^{7}$ The most common site of metastasis is the choroid, because of its rich vascularity. The choroid is a component of the uvea, along with the iris and ciliary body. Uveal metastasis of gastrointes- tinal malignancy was reported in $4 \%$ of cases. The prevalence of metastasis to the choroid was reported to be 838 (88\%) of 950 metastatic foci in the uvea. ${ }^{7}$ For initial diagnosis, choroidal metastatic tumor has to be differentiated from melanoma, the most common malignancy in the choroid. ${ }^{8}$ MRI of the orbits is useful in the differential diagnosis of choroidal mass. The metastatic tumor features a well-circumscribed subretinal mass appearing as an isointense signal on the T1-weighted image and as a hypointense signal on the T2-weighted image. ${ }^{9}$ The diagnostic efficacy of $\mathrm{N}$-isopropyl-p- $\left({ }^{123} \mathrm{I}\right)$ iodoamphetamine single-photon emission computed tomography $\left({ }^{123}\right.$ I-IMP SPECT) has been reported. ${ }^{10}$ Patients with orbital metastasis often have disseminated disease from a primary malignancy and a previous malignant disease. Therefore, we 

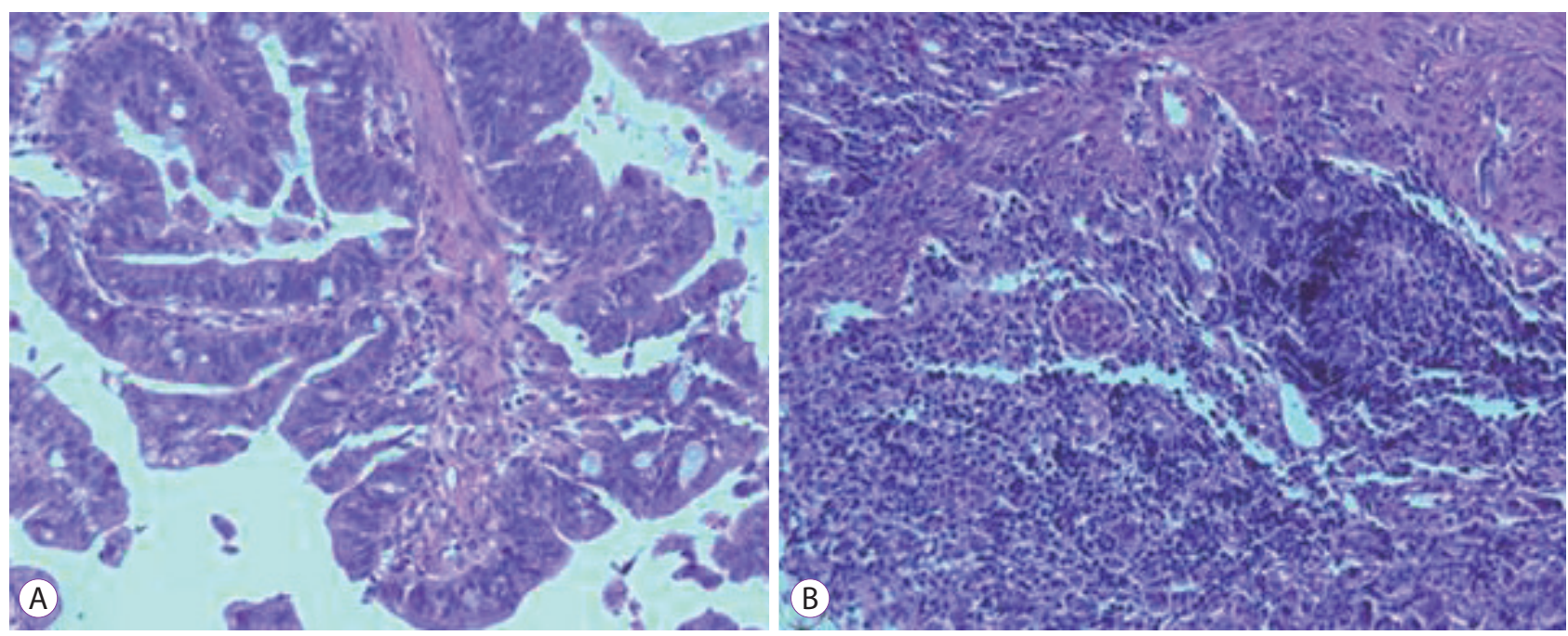

Fig. 4. (A) Endoscopic biopsy specimen from the stomach, showing moderately differentiated tubular adenocarcinoma (hematoxylin-eosin [H\&E] staining, original magnification $\times 100$ ). (B) Endoscopic biopsy specimen from the duodenum, showing a poorly differentiated tubular adenocarcinoma (H\&E staining, original magnification $\times 100$ ).

should consider systemic chemotherapy as the initial treatment option for patients with orbital metastasis. ${ }^{11-13}$

In addition, our case included small bowel metastasis. The incidence of small bowel cancer is $0.01 \%,{ }^{14}$ accounting for $0.35 \%$ of all malignancies. ${ }^{15,16}$ Among small bowel malignancies, metastatic lesion is more common than primary tumor. The most frequent site of duodenal metastases is the periampullary region, followed by the duodenal bulb. The most frequent presentations are gastrointestinal bleeding and anemia. ${ }^{17}$ The most common metastatic malignancy of the small bowel is melanoma, ${ }^{17,18}$ followed by lung cancer. ${ }^{19}$ For diagnosis of metastatic lesions, CT, barium study, and upper endoscopy with tissue sampling can be used. ${ }^{20}$

In summary, we report a rare case of advanced gastric cancer with systemic metastasis involving the intraocular choroid and duodenum at the initial diagnosis. In case of a patient with visual complaint and melena, the possibility of a metastatic malignancy should be considered.

\section{Conflicts of Interest}

The authors have no financial conflicts of interest.

\section{REFERENCES}

1. Feldman M, Friedman LS, Brandt LJ. Sleisenger and Fordtran's gastrointestinal and liver disease: pathophysiology, diagnosis, management. 9th Ed. Philadelphia (PA): Saunders/Elsevier; 2010.

2. Kong BD, Lee TW. Clinical analysis of metastatic intraocular malignancy. J Korean Ophthalmol Soc 1999;40:2928-2934.

3. Kim HJ, Kang JY. Metastatic gastric adenocarcinoma of the lower eyelid. J Korean Ophthalmol Soc 1995;36:1283-1286.

4. Kim JH, Yu HG. Clinical characteristics of metastatic choroidal tumors in Korean patients. J Korean Ophthalmol Soc 2008;49:1785-1793.
5. Eliassi-Rad B, Albert DM, Green WR. Frequency of ocular metastases in patients dying of cancer in eye bank populations. Br J Ophthalmol 1996;80:125-128.

6. Shields JA, Shields CL, Brotman HK, Carvalho C, Perez N, Eagle RC Jr. Cancer metastatic to the orbit: the 2000 Robert M. Curts lecture. Ophthal Plast Reconstr Surg 2001;17:346-354.

7. Shields CL, Shields JA, Gross NE, Schwartz GP, Lally SE. Survey of 520 eyes with uveal metastases. Ophthalmology 1997;104:1265-1276.

8. Tong KA, Osborn AG, Mamalis N, Harrie RP, Call NB. Ocular melanoma. AJNR Am J Neuroradiol 1993;14:1359-1366.

9. Peyster RG, Augsburger JJ, Shields JA, Hershey BL, Eagle R Jr, Haskin ME. Intraocular tumors: evaluation with MR imaging. Radiology 1988;168:773-779.

10. Goto H. Clinical efficacy of 123I-IMP SPECT for the diagnosis of malignant uveal melanoma. Int J Clin Oncol 2004;9:74-78.

11. Amer R, Peer J, Chowers I, Anteby I. Treatment options in the management of choroidal metastases. Ophthalmologica 2004;218:372-377.

12. Gragoudas ES. Current treatment of metastatic choroidal tumors. Oncology (Williston Park) 1989;3:103-110; discussion 111.

13. Kiratli H, Bilgiç S. Solitary choroidal metastasis managed by transpupillary thermotherapy. Eye (Lond) 2000;14 Pt 5:799-800.

14. Cusack JC, Tyler DS Jr. Small-bowel malignancies and carcinoid tumors. In: Berger DH, Feig BW, Fuhrman GM, eds. The MD Anderson surgical oncology handbook. Boston (MA): Little, Brown; 1995. p. 142-159.

15. Barclay TH, Schapira DV. Malignant tumors of the small intestine. Cancer 1983;51:878-881.

16. Michelassi F, Erroi F, Dawson PJ, et al. Experience with 647 consecutive tumors of the duodenum, ampulla, head of the pancreas, and distal common bile duct. Ann Surg 1989;210:544-554; discussion 554-546.

17. Kadakia SC, Parker A, Canales L. Metastatic tumors to the upper gastrointestinal tract: endoscopic experience. Am J Gastroenterol 1992;87:1418-1423.

18. Geboes K, De Jaeger E, Rutgeerts P, Vantrappen G. Symptomatic gastrointestinal metastases from malignant melanoma. A clinical study. J Clin Gastroenterol 1988;10:64-70.

19. McNeill PM, Wagman LD, Neifeld JP. Small bowel metastases from primary carcinoma of the lung. Cancer 1987;59:1486-1489.

20. Loualidi A, Spooren PF, Grubben MJ, Blomjous CE, Goey SH. Duodenal metastasis: an uncommon cause of occult small intestinal bleeding. Neth J Med 2004;62:201-205. 